\title{
Gouy phase shift measurement in a high finesse cavity by optical feedback frequency locking
}

\author{
Léo Djevahirdjian*1, Guillaume Méjean ${ }^{1}$, and Daniele Romanini ${ }^{1}$ \\ ${ }^{1}$ Université Grenoble Alpes, France
}

October 11, 2019

\begin{abstract}
We demonstrate a simple and robust scheme to measure the relative position of transverse cavity modes with high precision. This is based on robust optical-feedback frequency locking of a diode laser to a cavity resonance. A fraction of the laser power is then frequency shifted and used to re-inject other cavity resonances to obtain their positions relative to the locking resonance. This delivers the Gouy phase shift of the cavity modes with precision down to $6.4 \times 10^{-8} \mathrm{rad}$, comparable to previous state of the art based on a more complex setup. This should also be of interest for high precision/accuracy measurement of distance and displacement.
\end{abstract}

\section{Introduction}

In 1890, the french physicist Louis Georges Gouy was performing interference experiments and while observing fringe patterns he remarked that these changed when replacing a plane wave by a focused light beam $[1,2]$. The dark fringes became bright fringes and vice-versa, showing that a pi phase change was occurring with the focused beam. This is considered as the first report that a wave acquires a phase shift along its propagation axis when passing through a focal point. A first explanation can be obtained directly from Gaussian beam properties [3]. A more satisfying physical interpretation was proposed by Feng et al. [4] using the uncertainty principle associated to the spatial confinement of a focused wave in undulatory mechanics. More precisely, the Gouy phase shift is a consequence of the transverse momentum spread of photons as their trajectory is limited in the transverse direc- tion, which may result from focusing (in one or two dimensions) or from passing through a circular or slit aperture. The effect is therefore a manifestation of the uncertainty principle of Fourier transform theory applied to spatial waves. This explanation is universal in character, for instance it also applies to acoustic waves [5]. It is also quantitative, as it yields the correct result of the dependence of the Gouy phase shift for the Transverse Electro-Magnetic (TEM) modes of propagation of light [4].

The correct determination of the Gouy phase is relevant to various important applications such as laser wavelength metrology [6], precise distance measurement [7], accurate refractive index determination [8] and gravimetry [9].

To the best of our knowledge, state of the art measurements of Gouy phase shifts were performed by Durand et al., achieving a $2.7 \times 10^{-6} \mathrm{rad}$ accuracy and $6 \times 10^{-7} \mathrm{rad}$ pre-

*Corresponding author: leo.djevahirdjian@univ-grenoble-alpes.fr 
cision with $10 \mathrm{~s}$ measurements [10]. Their setup involved three narrow-linewidth fiber lasers independently locked to different resonances of a high finesse cavity $(\mathrm{F}=20000)$ by using the standard Pound-Drever-Hall (PDH) technique, requiring electro-optic modulators. They measured the beating frequencies between these locked lasers using fast $(20 \mathrm{GHz})$ photodiodes and frequency counters. We propose here a simpler approach using a single DFB diode laser and a simpler frequency locking scheme based on resonant optical feedback from the cavity. This technique is robust as it works even with DFB diode lasers possessing multi-MHz linewidth, while the $\mathrm{PDH}$ technique requires narrow line lasers (with short-term linewidth comparable to cavity resonances). For our implementation we use a $\mathrm{V}$-shaped cavity with finesse $\mathrm{F}=4300$, still we are able to improve on the precision of $\mathrm{Du}$ rand et al., down to $6.4 \times 10^{-8} \mathrm{rad}$ with $100 \mathrm{~s}$ averaging.

We should underline that the choice of a V-shaped cavity helps to simplify the optical feedback locking scheme but is not strictly necessary and does not reduce the generality of our approach. Indeed, optical feedback locking was exploited by Durand et al. [11] with a linear cavity to obtain record sensitivity in gas birefringence measurements, and by Hamilton et al. [12] with a ring cavity for spectroscopy and trace gas analysis.

We should also remark that optical feedback locking works well in the limit of very high finesse [11, 13]. Since the precision of frequency measurements would increase proportionally with the finesse, there is still much place for improvement just by using better mirrors. Main drawback would be the increased cavity response time required to obtain peak transmission signals and determine the center frequencies of cavity resonances.

\section{Theory}

Inside a stable optical resonator or cavity, light is focused at each round trip and acquires a Gouy phase shift. It is customary to consider the transverse electro-magnetic modes which constitute an orthonormal set of functions for expressing any light field propagating along an optical axis. When propagating inside an optical resonator, these $\mathrm{TEM}_{m, n}$ modes (with transverse order numbers $m, n$ ), acquire larger Gouy phase shifts proportionally to $m, n[14$, 15]. As we will see below, this translates into different resonance frequencies as a function of $m, n$. Measuring transverse modes frequencies gives then access to their Gouy phase shifts.

For a V-shaped cavity with equal mirrors (see fig. 1), which the curvature of the folding mirror renders astigmatic, the accumulated phase on one round-trip for a TEM cavity mode (assuming symmetrical arms) is found to be:

$$
\begin{aligned}
\phi_{m, n}= & 2 k L+\phi_{M} \\
& -4\left[\left(m+\frac{1}{2}\right) \phi_{G}^{x}+\left(n+\frac{1}{2}\right) \phi_{G}^{y}\right]
\end{aligned}
$$

where $k=2 \pi / \lambda=2 \pi \nu / c$ is the wave vector corresponding to wavelength $\lambda$ or optical frequency $\nu$ (through the speed of light in air $c)$, and $L$ is the total optical length of the cavity $\left(=2 L_{\text {arm }}\right.$ defined in fig. 1$)$. The term $\phi_{M}$ is the total dephasing due to reflections on the cavity mirrors for one cavity pass, which is in principle independent of the transverse mode order while it depends on polarization and frequency. This last dependence is weak inside the mirror working range, and we will neglect it (for a more complete discussion see $[10])$. The dependence on polarization, due to the off-normal reflection on the folding cavity mirror, is considered later.

The last term in eq. (1) is the expression of the accumulated Gouy phase taking into account the astigmatism of the cavity, which induces different confinement of light for the two transverse directions (relative to the cavity folding plane). Thus, there are different $\phi_{G}^{x}$ and $\phi_{G}^{y}$ Gouy phases along the horizontal $x$ and vertical $y$ axis (corresponding respectively to the transverse mode indices $m$ and $n$ ). Confusion should be avoided with the horizontal and vertical polarization states of light that are additionally allowed for any transverse mode. Polarization is actually exploited 
in the measurement procedure discussed later on. Finally, the factor 4 for the Gouy phases rather than a factor 2 as in the expression for a linear cavity, is due to the presence of 2 identical focal points inside the $\mathrm{V}$-shaped cavity, thus 4 passes through a focal point rather than just 2 .

As the resonance condition for the $N^{\text {th }}$ longitudinal cavity mode of a given transverse order is $\phi_{m, n}=2 \pi N$, eq. (1) gives:

$$
\begin{aligned}
\nu(N, m, n)=\mathrm{FSR}\left[N-\frac{\phi_{M}}{2 \pi}\right. & +\frac{2}{\pi}\left(\left(m+\frac{1}{2}\right) \phi_{G}^{x}\right. \\
& \left.\left.+\left(n+\frac{1}{2}\right) \phi_{G}^{y}\right)\right]
\end{aligned}
$$

where $\mathrm{FSR}=c / 2 L$ is the free spectral range of the cavity, that is, the separation of successive resonances with the same transverse order.

In particular, applying eq. (2) to calculate mode intervals, where the constant mirror dephasing $\phi_{M}$ cancels out, we can write:

$$
\begin{aligned}
\Delta \nu_{x} & =\nu(N, 1,0)-\nu(N, 0,0) \\
& =\frac{2}{\pi} \operatorname{FSR} \phi_{G}^{x} \\
\Delta \nu_{y} & =\nu(N, 0,1)-\nu(N, 0,0) \\
& =\frac{2}{\pi} \operatorname{FSR} \phi_{G}^{y}
\end{aligned}
$$

From this we finally express the astigmatic Gouy phases as a function of these measurable frequency intervals:

$$
\begin{aligned}
\phi_{G}^{x} & =\frac{\pi}{2} \frac{\Delta \nu_{x}}{\mathrm{FSR}} \\
\phi_{G}^{y} & =\frac{\pi}{2} \frac{\Delta \nu_{y}}{\mathrm{FSR}}
\end{aligned}
$$

\section{$3 \quad$ Experimental setup}

For accurate and stable measurements of frequency intervals between transverse cavity modes, we exploit resonant optical feedback frequency locking. This allows to keep the laser continuously locked to a given cavity mode while inducing a collapse of its linewidth, which becomes significantly narrower than the cavity resonance [16]. By resonant optical feedback we mean feedback produced by photons from a cavity resonance, thus spectrally filtered by the cavity. We use for this a V-shaped cavity geometry, as shown in fig. 1, which avoids feedback from the input mirror (in contrast to a linear cavity), while it allows feedback from photons which circulate inside the cavity at resonance [17]. In order to continuously maintain this locking condition it is necessary that the feedback wave from the cavity reaches the laser in phase with the emitted laser field. This is obtained by fine control of the position of one of the steering mirrors by a piezoelectric translator (pzt in fig. 1). An error signal for this correction is generated by applying a small modulation (at about $7 \mathrm{kHz}$ ) to the position of the same mirror, followed by lock-in detection on the cavity transmitted signal (from PD1 in fig. 1). We thus obtain an ultranarrow (sub-kHz) and stable laser source analogous to that described by Burkart et al. [18], with the difference that there the $\mathrm{V}$ shaped cavity is made out of ultra-low expansion glass installed inside a high vacuum thermally stabilized chamber. The cavity used here is made of massive aluminum, is installed inside a plexiglass enclosure, and its temperature is stabilized a few degrees above room temperature by using heating resistances. Residual temperature fluctuations are seen occur over timescales of about 2 hours and are bound to $40 \mathrm{mK}$ pk-pk, which (by considering thermal expansion coefficient of aluminum) translates to maximum frequency fluctuations of the cavity modes (around $764 \mathrm{~nm}$ ) by about $200 \mathrm{MHz}$. Correspondingly, the interval between mode frequencies, which we measure, is expected to suffer a drift not larger than about $200 \mathrm{~Hz}$.

According to fig. 1, we use a $764 \mathrm{~nm}$ DFB diode laser (Nanoplus GMBH) of vertical linear polarization, mode matched and frequency locked to a $\mathrm{V}$-shaped cavity with a vertex $32^{\circ}$ angle and a total length $L=468 \mathrm{~mm}$. The three mirrors are concave with a curvature radius of $500 \mathrm{~mm}$, they are wedged and have an anti-reflection coating on the back face of 
the substrate. They are pre-aligned and glued to removable aluminum mounts (allowing easy removal for cleaning) which are screwed on a aluminum block whose shape is suggested in fig. 1, where the two cavity channels have been drilled over a length of $233 \mathrm{~mm}$ and at an angle of $32^{\circ}$.

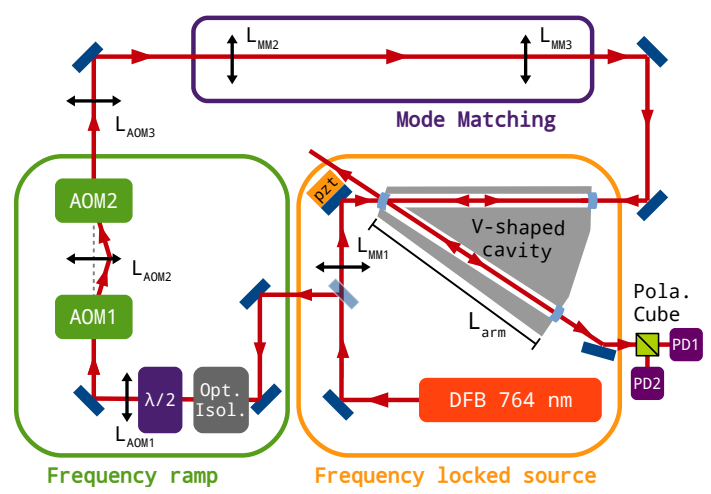

Figure 1: Experimental setup.

The diode laser is driven by a low noise current source working on batteries (ILX LDX3620) and by a high stability temperature controller (Thorlabs ITC502). Using optical feedback, the laser is locked at frequency $\nu_{\text {lock }}$ to a vertically polarized $\mathrm{TEM}_{0,0}$ cavity mode, $\mathrm{TEM}_{\text {lock }}$ in the following.

Part of the light $(70 \%)$ from this frequency-locked source is collected by a beamsplitter and sent through an optical isolator to avoid any parasite feedback that could perturb the optical lock.

The beam is then focused through a system composed of two Acousto-Optic Modulators (AOM) with 150-250 MHz working range, driven at a radio frequency $(\mathrm{RF}) \delta \nu_{A O M}$ from a waveform generator (240 MHz bandwidth), which allows to shift the optical frequency to $\nu_{\text {lock }}+2 \delta \nu_{A O M}$. These AOMs are mounted with a focusing lens at half distance between them, so that the first diffraction order of the first AOM is refocused on the second AOM mounted to have its first order deflected in the opposite direction. As the two AOMs are driven by the same RF source, the output of the second AOM provides exact compensation of the angular beam deviations of the first AOM, for any $\mathrm{RF}$ value in the AOM working range. We should underline that light polarization must arrive on the AOMs either parallel or perpendicular to their diffraction plane in order to avoid formation of an elliptical polarization state. A half-wave plate is therefore installed after the optical isolator in order to change its output $45^{\circ}$ linear polarization to horizontal.

The frequency shifted beam is modematched and injected to the V-shaped cavity through one of its end mirrors. In order to promote the excitation and thus the observation of transverse modes, the injected beam can be slightly shifted or tilted relative to the cavity axis. We underline that turning polarization to horizontal before cavity injection allows to excite cavity modes with polarization orthogonal to that of the cavity-locking mode and thus to easily separate cavity output signals of different origin by polarization. Using orthogonal polarization is also a key point to avoid perturbation of the optical feedback lock by light re-injected into the cavity (except for the $\mathrm{TEM}_{0,0}$ mode, as we will see later).

Light spilling out of the other cavity end mirror is analyzed by a polarizer cube, which allows monitoring vertical and horizontal polarization states on separate photodiodes and/or cameras. One of the photodiodes (PD2 in fig. 1) allows to obtain frequency profiles of horizontal cavity modes as the frequency shift is linearly tuned in time. On the other hand, the cameras allow to obtain the transverse mode pattern for easy identification of the transverse mode order.

\section{Measurement}

The cavity-locked DFB diode laser is used as a frequency reference which is stable relative to the cavity resonances during measurements. By changing the RF shift, the cavity re-injected laser beam can be made to resonate with any transverse mode on the horizontal polarization as long as this can be attained in the range allowed by the AOMs and the waveform generator (from 300 to $480 \mathrm{MHz}$ ). The 
transverse order is then readily deduced from the stable camera image observed at cavity output for the same polarization (see fig. 2).

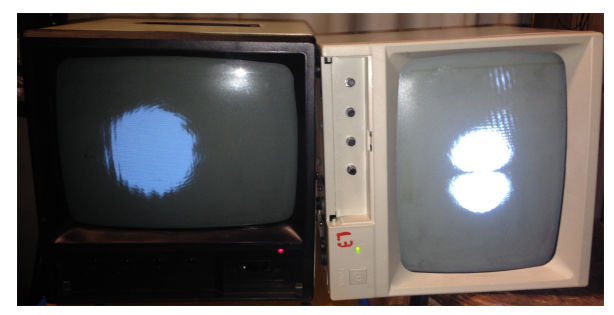

Figure 2: Output of the cavity for the vertical and horizontal polarizations, injected to different TEM cavity modes and monitored with two cameras.

In order to determine the frequency shift of the re-injected mode, a RF sweep of $400 \mathrm{kHz}$ around an initial rough guess of the resonance frequency is applied to the AOMs for a total sweep of $800 \mathrm{kHz}$, during typically $2 \mathrm{~ms}$. This linear sweep allows recording the transmission profile of the mode versus frequency (using a photodiode with response time $\sim 1 \mu \mathrm{s}$ ). The frequency accuracy and stability of our RF generator, which is referenced to a GPS disciplined $\mathrm{Rb}$ clock, is better than $10^{-10}$. The transmission profile is then digitized and fitted (using a Python routine based on the lmfit [19] library) by a Lorentzian function includ- ing a baseline term, to obtain a refined estimate of the resonant mode center frequency (fig. 3). From the modes linewidth $(\sim 75 \mathrm{kHz})$ we deduce a cavity finesse of about 4300 .

The scheme in fig. 4 illustrates the determination of the mode frequencies needed to calculate the $\Delta \nu$ quantities in eqs.(4). Given that the AOMs are used on their -1 order of diffraction and the limited frequency shift bandwith (300-480 MHz, we record the positions of the horizontally polarized $\mathrm{TEM}_{0,0}$, $\mathrm{TEM}_{0,1} / \mathrm{TEM}_{1,0}$ modes with longitudinal or$\operatorname{der}(N-1)$ and $(N-2)$ relative to the $\mathrm{TEM}_{\text {lock }}$ mode of order $N$ and vertical polarization. These positions are derived as stated above by sweeping the re-injected beam frequency around each mode. Profiles of the 3 modes are obtained in rapid succession: 100 scans of $2 \mathrm{~ms}$ over each mode, thus $\sim 700 \mathrm{~ms}$ measurement time (including time lags) to obtain 100 position determinations for each of the 3 modes. Each series of 100 position determinations are averaged before being used in eqs.(4). This allows to minimize the effect of cavity length fluctuations in between successive determinations of the mode frequencies.

Faster frequency switching could improve against short term effects (mechanical cavity vibrations) but would impose too much time lags in our measurements due to delays in the communication protocol of our RF generator.

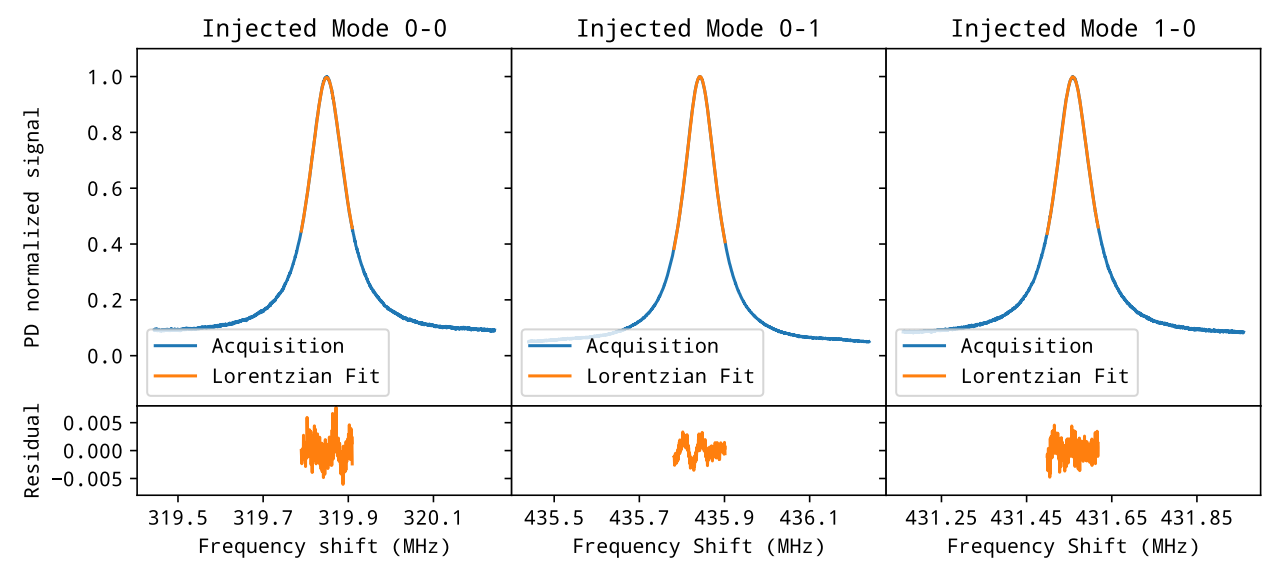

Figure 3: Acquired mode profiles (2 ms acquisition time) with fits and residuals. Different noise levels are due to different peak values before normalization. 


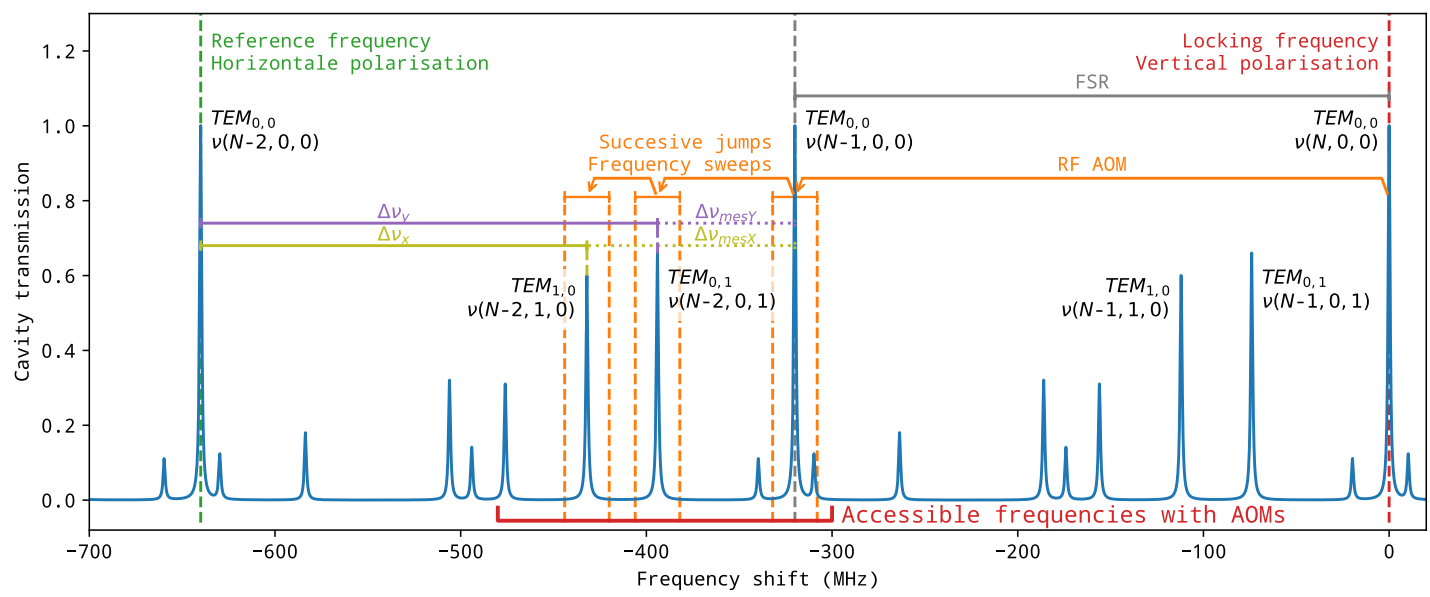

Figure 4: Measurement of $\Delta \nu$ values. AOMs RF range limitations impose to evaluate the frequency intervals relative to the end of the mode structure $\left(\Delta \nu_{m e s}\right.$ quantities). From there, the $\Delta \nu$ quantities are deduced using evaluation of FSR (in gray).

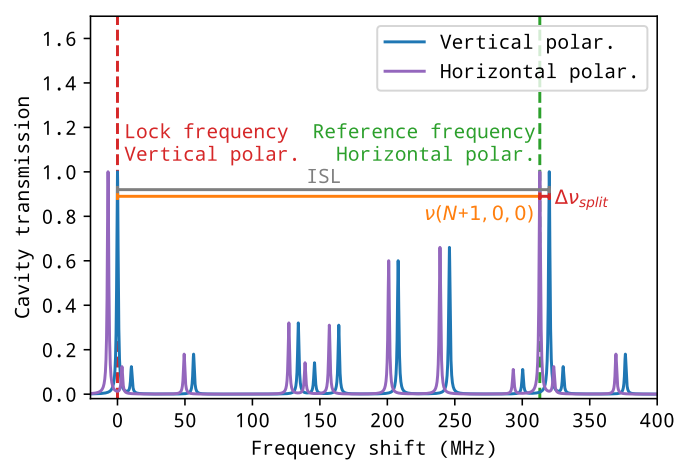

Figure 5: Measurement of FSR.

From there, the $\Delta \nu_{x}$ and $\Delta \nu_{y}$ quantities can be calculated using cavity FSR which is also required in eqs.(4). We obtain it from the position of the $(N-1) \mathrm{TEM}_{0,0}$ mode of horizontal polarization, corrected (see fig. 5) for the polarization mode splitting of the Vshaped cavity: $\mathrm{FSR}=\Delta \nu_{0,0(h)}-\Delta \nu_{\text {split }}$. This splitting is due to the off-normal reflection at the folding cavity mirror (the input mirror). It can be understood by considering the wellknown Fresnel equations [20, 21] for reflected $\mathrm{s}$ and $\mathrm{p}$ waves at a simple dielectric interface. These equations yield different phase shifts as a function of the incidence angle. Then, upon off-normal reflection on the alternate in- dex stack of a dielectric mirror, different phase delays are also accumulated by vertically and horizontally polarized waves. This phase shift translates into a splitting (independent of $m, n$ but slowly dependent on wavelength) of each $\mathrm{TEM}_{m, n}$ resonance according to polarization. In other words, in a V-shaped cavity each transverse mode comes as a frequency doublet with vertical and horizontal polarizations separated by an almost constant frequency gap $\Delta \nu_{\text {split }}$.

To determine the polarization splitting of our cavity, the polarization of the re-injected beam is turned to $45^{\circ}$, thanks to a second half-wave plate placed after the AOMs. This allows to obtain the $\mathrm{TEM}_{1,0}$ doublet profiles within a single $1.2 \mathrm{MHz}$ frequency scan over $2 \mathrm{~ms}$ : The vertically polarized component of the doublet appears on the signal from PD1 (with an offset given by the locked laser) while the other on the signal from PD2. In fact, when we tried to perform this type of measurement directly on the doublet of the $\mathrm{TEM}_{0,0}$ mode (with $N-1$ ), the laser lock was perturbed when scanning over the vertical polarized component. Instead, thanks to spatial orthogonality of different transverse modes (of same polarization), no perturbation was observed when using the 1,0 mode. Fi- 
nally, after fitting with a Lorentzian profile on a linear baseline, the center positions of the $\mathrm{TEM}_{1,0}$ modes of the two polarizations were subtracted to obtain a splitting value of $393471 \pm 5 \mathrm{~Hz}$ (from an average of $1.8 \times 10^{6}$ acquisitions over $1 \mathrm{~h}$ ).

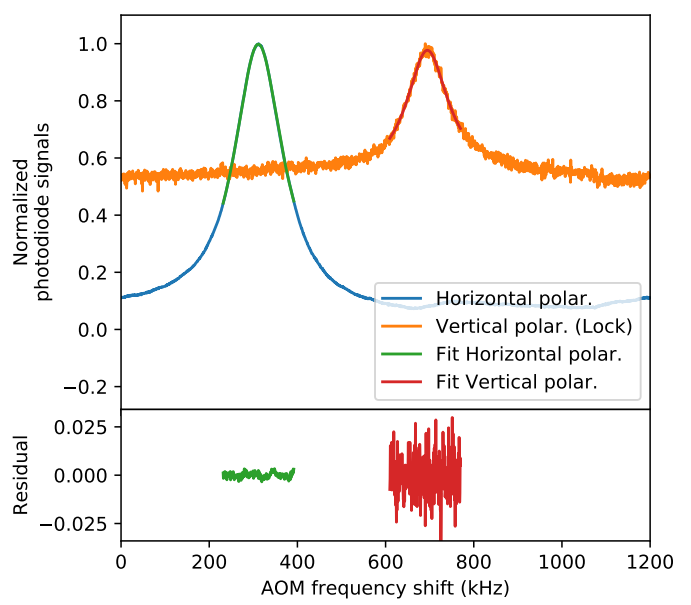

Figure 6: Measurement of the splitting.

It should be noted that this splitting, which depends on the incidence angle and the dielectric refraction indices of the coating materials of the mirror, is subject to significantly smaller thermal drift compared to the other frequency intervals contributing to our determination of the Gouy phases. This is comforted by the Allan deviation on this parameter which decreases steadily over the acquisition time mentioned above. Thus in practice, the measurement error on the splitting has negligible impact on the precision budget. On the other hand, its frequency dependence is not negligible: from modelling of the dielectric mirrors we estimate its value changes by about $140 \mathrm{~Hz}$ over one cavity FSR. Given that we measure the splitting not on the $(N-2)$ $\mathrm{TEM}_{0,0}$ mode (our virtual reference according to fig. 4) but on the $(N-1) \mathrm{TEM}_{1,0}$ mode, we introduce a systematic error of about $100 \mathrm{~Hz}$, which is here negligible relative to other systematic biases discussed below. On the other hand, this error could be completely avoided by addressing modes of the same polarization, which was not possible here given the limited $\mathrm{RF}$ range combined with the large cavity mode spacing.

\section{Results and discussion}

The stability of our simple locking system allows to average the mode positions during hours without interruption. In fig. 7, we show results from a 1-hour continuous acquisition set, where profiles of 3 transverse modes $\left(\mathrm{TEM}_{0,0}, \mathrm{TEM}_{0,1}\right.$ and $\left.\mathrm{TEM}_{1,0}\right)$ are acquired in succession, fitted, and resulting (averaged) mode frequencies are used to calculate the plotted quantities. The obtained traces for $\Delta \nu$ quantities show a doubling that probably corresponds to an oscillation on short time scales, probably an electronic artifact whose origin we still have to understand.

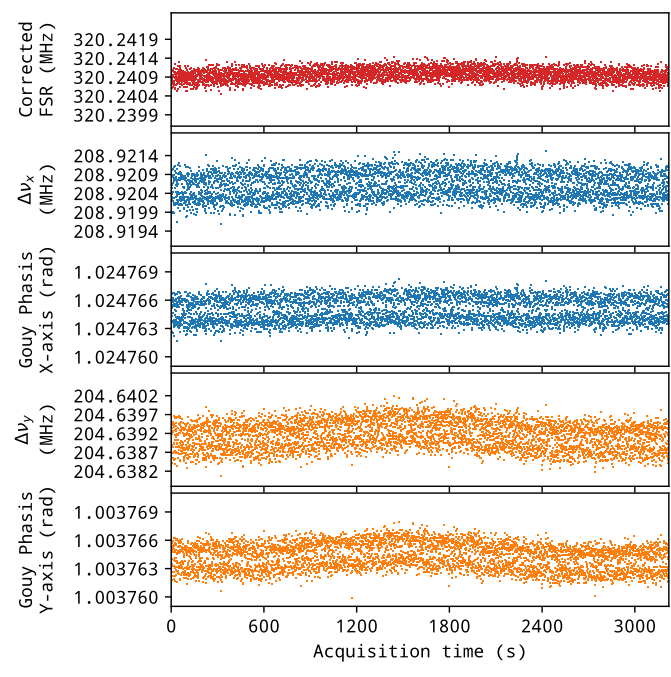

Figure 7: One set of measurements during 1 hour.

Shot to shot fluctuations of these quantities are governed by statistical type-A uncertainty, where averaging over $n$ successive samples delivers values with fluctuations decreasing as $1 / \sqrt{n}$. However, as the system is subject to drift, mostly of mechanical origin due to temperature changes, at long enough times this starts dominating over the statis- 
tical fluctuations of the averaged quantities. We will use the Allan variance [22] to study the effect of drift on our measurements.

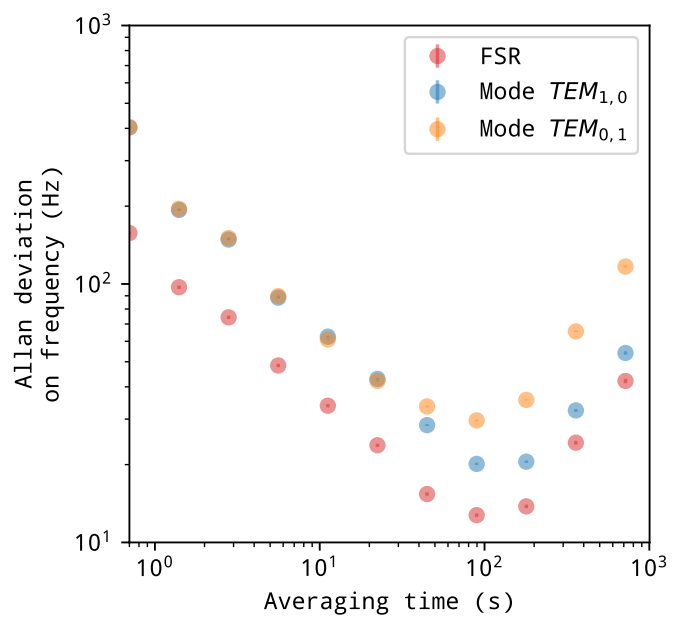

Figure 8: Allan deviations of mode positions.

Allan deviations (square-root of the variance) are calculated here with a Python routine using the allantools [23] library which delivers the "overlapping" Allan deviation [24].

In fig. 8 we see that frequency positions of modes relative to $\mathrm{TEM}_{\text {lock }}$ (mode intervals) are initially determined with few hundred $\mathrm{Hz}$ precision (for the $0.7 \mathrm{~s}$ acquisition time of an elementary measurement cycle). Averaging over $100 \mathrm{~s}$ leads to $10 \mathrm{~Hz}$ precision for the FSR determination, and a few times worse for the other mode intervals. This difference is mostly due to the better signal to noise ratio for the transmission peak obtained for the 0,0 mode. Further averaging up to 1 hour is clearly affected by drift degrading precision back up to the hundred $\mathrm{Hz}$ range. A similar trend is observed in fig. 9 for the Gouy phases calculated from these three frequency intervals. The minimum of the Allan deviation plot gives the lowest error achieved for the Gouy phases in our setup and with our measurement scheme. While this is about $1.2 \times 10^{-7} \mathrm{rad}$ for $\phi_{G}^{y}$, it goes down to $6.4 \times 10^{-8} \mathrm{rad}$ for $\phi_{G}^{x}$. This difference is probably due to the higher rigidity of our V-shaped cavity design on the horizontal plane.

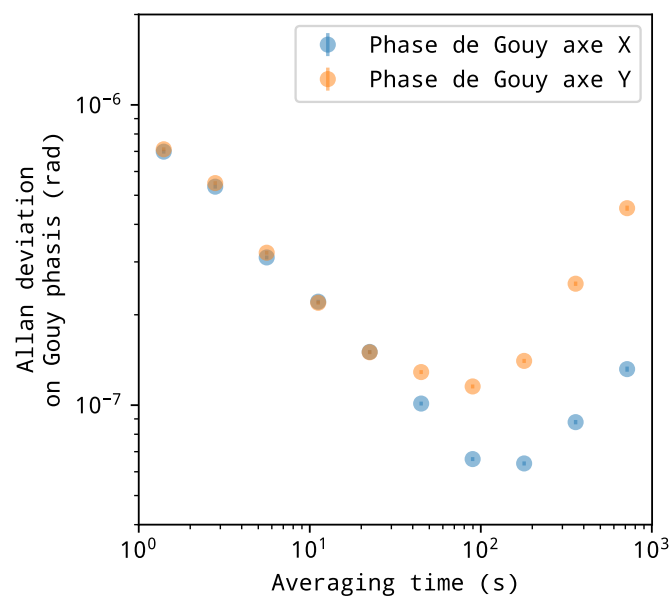

Figure 9: Allan deviations of Gouy phases.

We can attribute the observed behavior of the Allan deviation of the modes intervals to thermal cavity drift. As we observed in the experimental section, residual thermal drift of the cavity length after active thermal stabilization is limited in extent to $40 \mathrm{mK}$ peak to peak, which corresponds to a maximum modes interval change of about $200 \mathrm{~Hz}$, occurring over about 2 hours timescales. On the other hand, the observed Allan deviations start with few hundred $\mathrm{Hz}$ precision on short time scales, which is determined by signal to noise of fitted resonance profiles. Then Allan deviations decrease as precision improves with averaging until reaching the tens $\mathrm{Hz}$ range at two minutes. We may assume this minimum to be given by the thermal drift, which increases with time. However, if we naively take a linear drift in time by using a $100 \mathrm{~Hz}$ /hour slope, we should expect a ten $\mathrm{Hz}$ level to be attained on 6 minutes time scales. However, it is rather intuitive that drift on short time scales must be significantly faster than the slope given by the maximum deviation divided by the characteristic time scale over which it occurs. Thus we conclude that the minimum of the Allan deviation is consistent with cavity thermal drift. On the other hand, the longterm Allan deviation should go up to the expected maximum drift, which is the case here even though we did not measure the Allan de- 
viation at even longer time scales. In fact if we assume no other dominant drift mechanisms, we should expect a leveling off of the Allan variance at longer time scales. Thus it will be interesting in the future to use better temperature stabilization and/or longer acquisition times to verify this hypothesis.

With respect to systematic errors, besides the polarization splitting discussed above (negligible for the time being), we should consider the offset of the locked laser frequency relative to the center of the $\mathrm{TEM}_{\text {lock }}$ mode. As with other frequency locking schemes, this offset may be due either to parasitic etalon effects or to a non-zero locking point due to electronic drift or to residual amplitude modulation of the source as a result of applied modulation (of the feedback phase through the piezoelectrically controlled mirror). The entity of changing frequency offset can be roughly evaluated by looking at variations in the cavity transmission level for the vertical polarization (lock) on the PD1 photodiode. By considering the width of the cavity resonances, for our measurements these variations can be translated to a maximum offset of $\pm 2 \mathrm{kHz}$, corresponding to a maximum $\pm 12 \times 10^{-6}$ rad error on the Gouy phases. This estimate is likely in large excess since changes in the cavity transmission level may have several origins besides drift of the locking point.

While we could find a way to measure and control this locking frequency offset, we think it would be preferable to circumvent it altogether. Thus an interesting improvement would be to use AOMs with wider bandwidth or a cavity with a smaller FSR. This would allow to perform direct measurement of FSR and other $\Delta \nu$ values relative to the $\mathrm{TEM}_{0,0}$ mode of the same polarization, by including this mode in our rapid measurement cycle. Such differential measurements would free our measurements from any offset of the locking frequency (as long as this is slowly drifting) and also from the need to consider the polarization splitting.

Finally, we should observe that the measured Gouy phases are consistent with expected values for our cavity, and can be made to coincide to within measurement accuracy by small adjustment of the radius of curvature $R$ of the three mirrors (for simplicity supposed identical) and of the cavity folding angle. For this check, we applied the standard Gaussian beam propagation model based on transfer matrices for the cavity mirrors and the freespace propagation in between them. Astigmatism is introduced by the incidence angle $\theta$ on the folding mirror which gives two different mirror matrices [25] for propagation in the horizontal and vertical planes. As discussed e.g. by Yariv[14], the round-trip Gaussian beam propagation matrix can be used to calculate all geometrical properties of the cavity, in particular the TEM modes frequencies and the Gouy phases. We first set the cavity length to produce the measured FSR $(320.2410 \mathrm{MHz}$ from fig. 7), giving $L=234.1986 \mathrm{~mm}$ rather than $234.2 \mathrm{~mm}$ by construction. Then, by fitting $R$ and $\theta$ to obtain the Gouy phase values $\phi_{x}=1.024765 \mathrm{rad}$ and $\phi_{y}=1.003764 \mathrm{rad}(\mathrm{also}$ from fig. 7) we obtain respetively $496.667 \mathrm{~mm}$ (manufacturer specification is $500 \mathrm{~mm}$ ) and $15.6524^{\circ}\left(16^{\circ}\right.$ by construction). This is not surprising since two model parameters are adjusted to obtain perfect match for two dependent variables. In place of adjusting the cavity angle, we could also have allowed for mirror astigmatism by using different curvature radii in the vertical and horizontal planes. However, the message is that the adjusted parameter values do match the cavity design values to within reasonable mechanical tolerances.

\section{Conclusions}

To our knowledge, the best measurement of a Gouy phase in a cavity was made by Durand et al. [10] in 2012. They managed to achieve a $2.7 \times 10^{-6} \mathrm{rad}$ accuracy and $6 \times 10^{-7} \mathrm{rad}$ precision with only $10 \mathrm{~s}$ averaging (they did not provide Allan variance plots), using a $5 \times$ higher finesse cavity than here, and a more complex setup.

By using optical frequency locking we are able to reach similar performance for a much lower finesse cavity, with $6.4 \times 10^{-8} \mathrm{rad}$ pre- 
cision in $100 \mathrm{~s}$ averaging (and $2 \times 10^{-7} \mathrm{rad}$ in $10 \mathrm{~s}$ ). As our setup does not allow variation of the cavity length, we cannot present a thorough comparison of measurements with the theory as Durand et al. did. However, we estimate systematic errors and their drift as being not larger than $\pm 2 \mathrm{kHz}$ in the determination of the frequency intervals between modes, yielding $\pm 12 \times 10^{-6} \mathrm{rad}$ accuracy on the Gouy phases.

Our approach can be applied to linear or ring cavities as well, and higher finesse should not be a problem [11-13]. This should allow sensitivity increase of a few orders of magnitude, at the price of increased measurement time. An important improvement is to redesign our setup to allow sampling the cavity FSR on successive transverse modes of the same polarization, which would allow to set aside the polarization mode splitting and the associated frequency dependence (which may introduces a sizable bias). One could also use a more agile RF generator in order to reduce the commutation time between the resonances needed for the determination of mode intervals. The measurement scheme could also be adapted to allow measurement of distances or displacements within a cavity of adjustable size, in particular by using electrooptically generated sidebands in place of AOM frequency shifts, together with a more broadly tuneable RF generator.

\section{Acknowledgements}

This work would have not been possible without the precision mechanics from the machine shop of our laboratory (LiPHY, CNRS Grenoble). The DFB diode laser was a courtesy of the AP2E company (www.AP2E.com). L. D. acknowledges a Ph.D. fellowship by the Grenoble University AGIR program.

\section{References}

[1] L. G. Gouy. "Sur une propriété nouvelle des ondes lumineuses". In: Comptes rendus hebdomadaires des séances de
l'Académie des sciences (1890), p. 5 (cit. on p. 1).

[2] L. G. Gouy. "Sur la propagation anomale des ondes". In: Comptes rendus hebdomadaires des séances de l'Académie des sciences (1891), p. 5 (cit. on p. 1).

[3] R. W. Boyd. "Intuitive explanation of the phase anomaly of focused light beams". In: Journal of the Optical Society of America 70.7 (July 1, 1980), p. 877 . DOI: $10.1364 /$ JOSA .70 .000877 (cit. on p. 1).

[4] S. Feng and H. G. Winful. "Physical origin of the Gouy phase shift". In: Optics Letters 26.8 (Apr. 15, 2001), p. 485. DOI: 10.1364/OL.26.000485 (cit. on p. 1).

[5] N. C. R. Holme, B. C. Daly, M. T. Myaing, and T. B. Norris. "Gouy phase shift of single-cycle picosecond acoustic pulses". In: Applied Physics Letters (2003), p. 4 (cit. on p. 1).

[6] J.-P. Monchalin, M. J. Kelly, J. E. Thomas, N. A. Kurnit, A. Szöke, F. Zernike, P. H. Lee, and A. Javan. "Accurate laser wavelength measurement with a precision two-beam scanning Michelson interferometer". In: Applied Optics 20.5 (Mar. 1, 1981), p. 736. DOI: 10. 1364/A0.20.000736 (cit. on p. 1).

[7] I. Coddington, W. C. Swann, L. Nenadovic, and N. R. Newbury. "Rapid and precise absolute distance measurements at long range". In: Nature Photonics 3.6 (June 2009), pp. 351-356. DOI: 10. $1038 /$ nphoton . 2009.94 (cit. on p. 1).

[8] P. Kužel, H. Němec, F. Kadlec, and C. Kadlec. "Gouy shift correction for highly accurate refractive index retrieval in time-domain terahertz spectroscopy". In: Optics Express 18.15 (July 19, 2010), p. 15338. DOI: $10.1364 /$ OE.18.015338 (cit. on p. 1). 
[9] L. Robertsson. "On the diffraction correction in absolute gravimetry". In: Metrologia 44.1 (Feb. 2007), pp. 35-39. DOI: 10 . 1088 / 0026 - 1394 / 44 / $1 / 005$ (cit. on p. 1).

[10] M. Durand, Y. Wang, and J. Lawall. "Accurate Gouy phase measurement in an astigmatic optical cavity". In: Applied Physics B 108.4 (Sept. 2012), pp. 749753. DOI: $10.1007 / \mathrm{s} 00340-012-5147-\mathrm{x}$ (cit. on pp. 2, 9).

[11] M. Durand, J. Morville, and D. Romanini. "Shot-noise-limited measurement of sub-parts-per-trillion birefringence phase shift in a high-finesse cavity". In: Physical Review A 82.3 (Sept. 14, 2010), p. 031803. DOI: 10 . 1103 / PhysRevA . 82.031803 (cit. on pp. 2, 10).

[12] D. J. Hamilton, M. G. D. Nix, S. G. Baran, G. Hancock, and A. J. Orr-Ewing. "Optical feedback cavityenhanced absorption spectroscopy (OFCEAS) in a ring cavity". In: Applied Physics B 100.2 (Aug. 2010), pp. 233242. DOI: $10.1007 / \mathrm{s} 00340-009-3811-6$ (cit. on pp. 2, 10).

[13] J. Landsberg, D. Romanini, and E. Kerstel. "Very high finesse optical-feedback cavity-enhanced absorption spectrometer for low concentration water vapor isotope analyses". In: Optics Letters 39.7 (Apr. 1, 2014), p. 1795. DOI: 10.1364/ OL. 39.001795 (cit. on pp. 2, 10).

[14] A. Yariv. Quantum Electronics - Third Edition. John Wiley and Sons, 1975 (cit. on pp. 2, 9).

[15] D. Romanini. "Modelling the excitation field of an optical resonator". In: Applied Physics B 115.4 (June 2014), pp. 517531. DOI: $10.1007 / \mathrm{s} 00340-013-5632-\mathrm{x}$ (cit. on p. 2)

[16] P. Laurent, A. Clairon, and C. Breant. "Frequency noise analysis of optically self-locked diode lasers". In: IEEE Journal of Quantum Electronics 25.6 (June 1989), pp. 1131-1142. DOI: 10.1109/3. 29238 (cit. on p. 3).
[17] J. Morville, S. Kassi, M. Chenevier, and D. Romanini. "Fast, low-noise, mode-bymode, cavity-enhanced absorption spectroscopy by diode-laser self-locking". In: Applied Physics B 80.8 (June 2005), pp. 1027-1038. DOI: $10.1007 /$ s00340005-1828-z (cit. on p. 3).

[18] J. Burkart, D. Romanini, and S. Kassi. "Optical feedback stabilized laser tuned by single-sideband modulation". In: $O p$ tics Letters 38.12 (June 15, 2013), p. 2062. DOI: $10.1364 /$ OL . 38.002062 (cit. on p. 3).

[19] M. Newville and T. Stensitzki. lmfit, Non-Linear Least-Squares Minimization and Curve-Fitting for Python. 2014 (cit. on p. 5).

[20] A. Fresnel. "Mémoire sur la réflexion de la lumière". In: Mémoires de l'Académie (royale) des sciences de l'Institut (imperial) de France. 20 (1819), p. 195 (cit. on p. 6).

[21] A. Fresnel. "Mémoire sur la loi des modifications que la réflexion imprime à la lumière polarisée". In: Mémoires de l'Académie (royale) des sciences de l'Institut (imperial) de France. 11 (1823), p. 393 (cit. on p. 6).

[22] D. W. Allan. "Statistics of atomic frequency standards". In: Proceedings of the IEEE 54.2 (1966), pp. 221-230. DOI: 10.1109/PROC.1966. 4634 (cit. on p. 8).

[23] A. Wallin, D. Price, C. Carson, and F. Meynadier. AllanTools, a python library for calculating Allan deviation and related time $\&$ frequency statistics under LGPL v3+ license. 2014 (cit. on p. 8).

[24] J. J. Snyder. "An Ultra-High Resolution Frequency Meter". In: Thirty Fifth Annual Frequency Control Symposium. Thirty Fifth Annual Frequency Control Symposium. IEEE, 1981, pp. 464-469. DOI: 10.1109/FREQ.1981.200513 (cit. on p. 8).

[25] A. Siegman. "Ray Optics and Ray Matrices". In: Lasers. University Science Books, 1986, p. 585 (cit. on p. 9). 